\title{
Diffusion Coefficient and Solvation Free energy of Sucrose in Water: A Molecular Dynamics Study
}

\section{Sapkota and N. P. Adhikari}

Journal of Nepal Physical Society

Volume 7, Issue 4, December 2021

ISSN: 2392-473X (Print), 2738-9537 (Online)

JNPS

ISSN:2392-473X (Print) 2738-9537 (Online)

Journal of

Nepal Physical Society Volume 7, Issue 4, December 2021

\author{
Editors: \\ Dr. Binod Adhikari \\ Dr. Bhawani Joshi \\ Dr. Manoj Kumar Yadav \\ Dr. Krishna Rai \\ Dr. Rajendra Prasad Adhikari \\ Mr. Kiran Pudasainee
}

JNPS, 7 (4), 1-9 (2021)

DOI: http://doi.org/10.3126/jnphyssoc.v7i4.42924

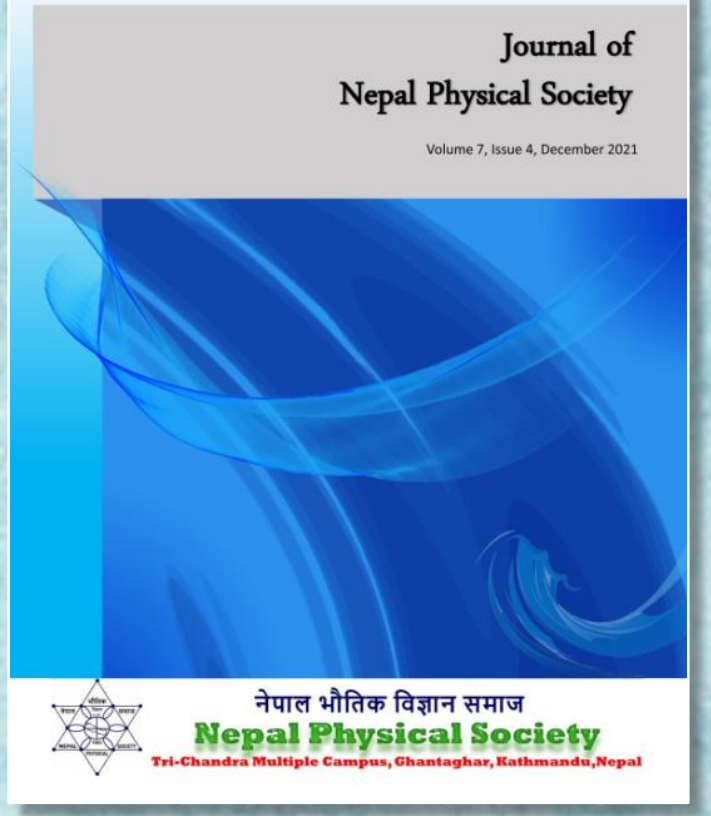

Published by:

Nepal Physical Society

P.O. Box: 2934

Tri-Chandra Campus

Kathmandu, Nepal

Email: nps.editor@gmail.com 


\title{
Diffusion Coefficient and Solvation Free energy of Sucrose in Water: A Molecular Dynamics Study
}

\author{
D. Sapkota ${ }^{1}$ and N. P. Adhikari ${ }^{2, *}$ \\ ${ }^{1}$ Golden Gate International College, Kathmandu, Nepal \\ ${ }^{2}$ Central Department of Physics, Kathmandu, Nepal \\ *Corresponding Email: narayan.adhikari@cdp.tu.edu.np
}

Received: 12th November, 2021; Revised: 5th December, 2021; Accepted: 25th December, 2021

\begin{abstract}
In this work, we have carried out Molecular Dynamics Simulation technique to study the diffusion coefficients, radial distribution functions and solvation free energy of sucrose $\left(\mathrm{C}_{12} \mathrm{H}_{22} \mathrm{O}_{11}\right)$ at different temperatures ranging from $298.15 \mathrm{~K}$ to $318.15 \mathrm{~K}$. We have taken 2 molecules of sucrose in 1343 molecules of extended simple point charge (SPC/E) water model. The self-diffusion coefficients are obtained by applying linear best fit to the mean square displacement (MSD) plot and binary diffusion coefficients are obtained by using Darken's relation. Arrhenius equation has been used to show a linear relationship between natural logarithm of binary diffusion coefficient and reciprocal of temperature. The structural analysis of the solution has been carried out with the help of radial distribution functions (RDFs) of its constituents. The solvation free energy of sucrose is studied at $300 \mathrm{~K}$ by using free energy perturbation method like Bennett Acceptance Ratio (BAR). The results of binary diffusion coefficients obtained from this simulation work agree very well with the previously reported experimental data.
\end{abstract}

Keywords: Sucrose; Molecular Dynamics; Diffusion Coefficient; RDF; Solvation Free Energy.

\section{INTRODUCTION}

The most abundant biomolecules on earth are carbohydrates which yield polyhydroxy aldehyde or ketone on hydrolysis. Three major types of carbohydrates: monosaccharides, oligosaccharides, and polysaccharides can be found in nature [1]. Sucrose, commonly known as sugar, is an example of oligosaccharide which is composed of $\alpha$-D glucose and $\beta$-D fructose residues. These residues are joined by an $\alpha, \beta$-glycosidic bond as shown in figure (1). The IUPAC name of sucrose is $\alpha$-D glucopyranosyl $\beta$-D- fructofuranoside and has molar mass $342.3 \mathrm{~g} / \mathrm{mol}$ [2]. It is highly soluble in water due to its eight hydroxyl groups which can form hydrogen bonds. Another significant and distinguishable property is sweetness. Its sweetness intensity is highest at $\sim 32.38^{\circ} \mathrm{C}$ [3]. It reported to have world's highest production of any single, pure, natural, organic chemical [4]. During digestive process, sucrose is released into glucose and fructose, which reach the liver via the portal vein, and they go into blood stream in the form of glucose. It has been observed that consumption of sucrose improves mental alertness, memory, reaction time, attention, ability to solve mathematical problems, and reduction in the feeling of fatigue [5]. However, some recent reports have also suggested that increased intake of sugar has adverse effects on cardiovascular health, increases blood pressure and can put life in risk [6]. Therefore, sucrose is the most important carbohydrates found in nature and thus, study of its transport properties has become an essential work for many researchers. The transport phenomena include fluid transport, heat transfer, and mass transfer. The mass transfer is the case of diffusion. Diffusion is the process by which mass is transferred from one part of a system to another as a result of random molecular motions [7]. The study of diffusion coefficient of sucrose is important both in nature and in technological applications. During metabolism of organisms, as 
energy source and structural agents, sucrose plays an important role and can protect from freezing or dehydration in extreme environmental conditions. Sucrose also has some important

technological applications in food preservation and in the cryopreservation of proteins [8]. In theoretical aspects of physics, the diffusion coefficient of sucrose in water can be used to estimate the various parameters such as the hydrodynamic radii and activation energy for the diffusion of aqueous sucrose. In addition, the measured diffusion coefficients can also be used to estimate activity coefficients [9]. Diffusion describes the dissolution and solubility phenomena, which are fundamental terms describing the process of solvation. They are related by the NoyesWhitney equation [10].

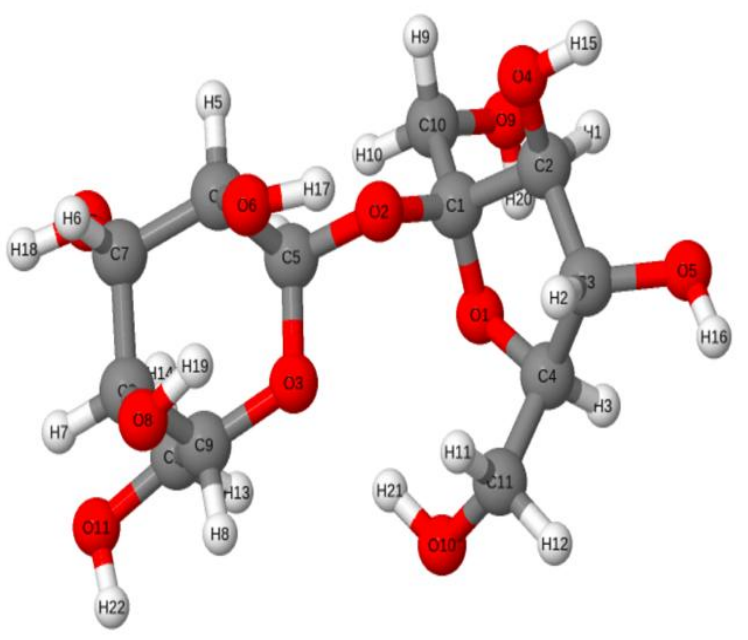

Fig.1: Three-dimensional structure of Sucrose.

Solvation is the phenomenon of transferring a solute from an ideal gas state into a liquid solution. The free energy change associated with this phenomenon is solvation free energy [11]. Finding free energy change of a physical system has a great interest in many fields, from drug design to basic statistics, to even non perturbative quantum chromodynamics [12]. To carry out computation of diffusion coefficient and solvation free energy of sucrose in water, Groningen Machine for Chemical Simulation (GROMACS) package is found to be very useful as it is user friendly and convenient to use with minimum expenditure of time. The scope of GROMACS is not only limited to calculation of diffusion coefficient or solvation free energy, but it can also calculate other physical macroscopic properties of a system like viscosity, thermal conductivity, electrical conductivity, radial distribution function, and activation energy [13].

\section{A. Diffusion Coefficient}

The macroscopic law that describes diffusion is known as Fick's law, which states that the flux $\boldsymbol{j}$ (rate of transfer per unit area) of the diffusing species is proportional to the negative gradient in the concentration of that species [14]:

$$
\boldsymbol{j}=-D \boldsymbol{\nabla} c(\boldsymbol{r}, t)
$$

where $D$, the constant of proportionality, is referred to as the diffusion coefficient (or self-diffusion coefficient) and $c(\boldsymbol{r}, t)$ is the concentration of the diffusing species at position $\boldsymbol{r}$ and time t. The negative sign indicates that the diffusion occurs in the direction opposite to that of increasing concentration. The famous Einstein's equation for self-diffusion coefficient is [15]

$$
D=\lim _{t \rightarrow \infty} \frac{1}{6} \frac{\partial<r^{2}>}{\partial t^{2}}
$$

$\left\langle r^{2}\right\rangle$ is the mean-squared displacement of the diffusing particle.

The binary diffusion coefficient between two diffusing particles in a binary mixture is given by Darken's relation [16]

$$
D_{12}=N_{1} D_{2}+N_{2} D_{1}
$$

$N_{1}, N_{2}$ are the mole fractions and $D_{1}, D_{2}$ are the self-diffusion coefficients of the mixing particles 1 and 2 in the mixture respectively.

\section{B. Solvation free energy}

We couple our solute molecule into the solvent through different intermediate states using coupling parameter $\lambda$. Taking enough intermediate states between initial ( $\lambda=0$; solute in gaseous state) and final $(\lambda=1 ;$ solute completely merged into solvent) states would give a useable value of free energy change since they have sufficient overlapping of phase space integrals [17]. For $N$ intermediate states, the free energy change $\left(\Delta G_{i j}\right)$ is computed by using the exponential average of the difference in the potential energies $\left(\Delta U_{i j}\right)$ between two consecutive states $i$ and $j$ [18]

$$
\Delta G_{i j}=-k_{B} T \frac{1}{N} \sum_{n=1}^{N} e^{-\beta \Delta U\left(x_{n}\right)_{i j}}
$$

$k_{B}$ is the Boltzmann constant, $T$ is the temperature, and $\beta=\frac{1}{k_{B} T}$.

This is Exponential Averaging (EXP) method. It depends on the direction of transformation i.e., measurement of $\Delta U_{i j}$ in the direction of increasing or decreasing entropy. To overcome this directional 
issue, Bennett Acceptance Ratio concept was introduced, which considers both directions of entropy. The total free energy change between two states A and B with $N$ intermediate states is [19]

$$
\begin{gathered}
\Delta G_{A B}=\frac{1}{\beta} \frac{\sum_{i=1}^{N_{1}} \frac{1}{1+e^{-\beta\left(\Delta U_{i}^{1}-C\right)}}}{\sum_{j=1}^{N_{0}} \frac{1}{1+e^{-\beta\left(\Delta U_{j}^{0}-C\right)}}}+C-\frac{1}{\beta} \ln \left(\frac{N_{1}}{N_{0}}\right) \\
C=\Delta G_{A B}+\frac{1}{\beta} \ln \left(\frac{N_{1}}{N_{0}}\right)
\end{gathered}
$$

$\Delta U_{i}^{1}$ and $\Delta U_{j}^{0}$ are the potential energy differences for configurations sampled from states 1 and 0 respectively.

\section{METHODOLOGY}

\section{A. Modeling of the system}

The modeling of the system constitutes two things: the interactions among the molecules making up the system and the interactions between the molecules and their environment. The molecular interactions are contained in an intermolecular force law or, equivalently, an intermolecular potential energy function whereas the interaction of molecules with the environment includes the boundary conditions [20]. The expression for the potential energy $U(r)$ of a molecular system is the linear contributions of bonded interactions coming from bond stretching $U_{b o n d}$, bond angle bending $\left(U_{\text {angle }}\right)$, bond dihedral $U_{\text {dihedral }}$, bond improper $U_{\text {improper, }}$ and nonbonded interactions coming from van der Waals potential (Lennard-Jones potential) $U_{v d W}$, and coulomb potential $U_{\text {coulomb }}$. Mathematically [21-23],

$$
U_{\text {bond }}=\sum_{\text {bonds }} \frac{K_{d}}{2}\left(d-d_{0}\right)^{2}
$$

$K_{d}$ is the force constant of the bond, $d_{0}$ is the equilibrium bond length between two atoms in bond. It is the bond length when all other potential energy terms are zero e.g., harmonic oscillator in vacuum.

$$
U_{\text {angle }}=\sum_{\text {angles }} \frac{K_{\theta}}{2}\left(\theta-\theta_{0}\right)^{2}
$$

$K_{\theta}$ is the force constant of angle vibration, $\theta_{0}$ is an equilibrium angle between three atoms.

$$
U_{\text {dihedral }}=\sum_{\text {dihedral }} \frac{K_{\phi}}{2}\left(1+\cos \left(n \phi-\phi_{0}\right)\right)
$$

$K_{\phi}$ is the barrier height, $n$ is the number of minima in the energy function, which is also called as multiplicity and $\phi_{0}$ is the phase factor.

$$
U_{\text {improper }}=\sum_{\text {improper }} \frac{K_{\psi}}{2}\left(\psi-\psi_{0}\right)^{2}
$$

$K_{\psi}$ is force constant of bond improper and $\psi_{0}$ is an equilibrium improper dihedral angle. This potential is introduced to preserve planarity of groups with flat geometry, and sometimes chirality of certain groups.

$$
\begin{gathered}
U_{v d W}=\sum_{\text {non-bonded pairs }(i, j)} 4 \epsilon_{i j}\left[\left(\frac{\sigma_{i j}}{r_{i j}}\right)^{12}\right. \\
\left.-\left(\frac{\sigma_{i j}}{r_{i j}}\right)^{6}\right] \\
U_{\text {coulomb }}=\sum_{\text {non-bonded pairs }(i, j)} \frac{q_{i} q_{j}}{4 \pi \epsilon_{D} r_{i j}}
\end{gathered}
$$

$\sigma_{i j}=\frac{1}{2}\left(\sigma_{i i}+\sigma_{j j}\right)$ is the collision diameter; $\epsilon_{i j}=\left(\epsilon_{i i} \epsilon_{j j}\right)$ is the potential well depth; $r_{i j}$ is the cartesian distance between two atoms $i$ and $j ; q_{i}$ and $q_{j}$ are the partial charges of a pair of atoms $(i, j)$; and $\epsilon_{D}$ is the dielectric permittivity of the medium. The bonded and non-bonded parameters for SPC/E water are shown in tables 1 and 2 respectively. These are taken from the data provided by GROMACS software.

Table 1: Bonded parameters for the SPC/E water; $\left(d_{0}\right)_{O H}$ is the equilibrium bond length between oxygen and hydrogen atoms $(\mathrm{O}-\mathrm{H}),\left(K_{d}\right)_{O H}$ is the force constants for the $\mathrm{O}-\mathrm{H}$ bond, $\theta_{0}$ is the equilibrium $\mathrm{HOH}$ bond angle, and $\left(K_{\theta}\right)_{\mathrm{HOH}}$ is the force constant of the bond angle vibration in water molecules.

\begin{tabular}{llll}
\hline$\left(K_{d}\right)_{O H}$ & $\mathbf{3 . 4 5 0 0} \times \mathbf{1 0}^{\mathbf{5}} \mathbf{k J m o l}^{-\mathbf{1}} \mathbf{n m}^{-2}$ & $\left(d_{0}\right)_{O H}$ & $0.1000 \mathrm{~nm}$ \\
\hline$\left(K_{\theta}\right)_{\mathrm{HOH}} \mathbf{3 . 8 3 0 0} \times \mathbf{1 0}^{2} \mathbf{k J m o l}^{-1} \mathbf{r a d}^{-2}$ & $\theta_{0}$ & $109.47^{0}$ \\
\hline
\end{tabular}

Table 2: Non-bonded parameters for oxygen (O1) of sucrose and oxygen (OW) of SPC/E water.

\begin{tabular}{llll}
\hline $\boldsymbol{\sigma}_{\boldsymbol{O} 1-\boldsymbol{O} 1}$ & $0.2900 \mathrm{~nm}$ & $\boldsymbol{\epsilon}_{\boldsymbol{O} 1-\boldsymbol{O} 1}$ & $0.59 \mathrm{~kJ} / \mathrm{mol}$ \\
\hline $\boldsymbol{\sigma}_{\boldsymbol{O W}-\boldsymbol{O W}}$ & $0.3165 \mathrm{~nm}$ & $\boldsymbol{\epsilon}_{\text {OW-OW }}$ & $0.65 \mathrm{~kJ} / \mathrm{mol}$ \\
\hline
\end{tabular}




\section{B. Simulation Procedure}

For both diffusion and free energy change processes, two molecules of sucrose are kept in a cubical box of size $3.5 \mathrm{~nm}$ length with 1343 molecules of SPC/E water. We are choosing SPC/E model over all other well-known models [24]. One of the reasons is that this model has a dipole moment of $2.35 \mathrm{D}$ and a polarization correction to the total electrical energy of $5.52 \mathrm{~kg} / \mathrm{mol}$, which is better as compared to other models [25]. The sucrose molecules are separated by $(0.5 \mathrm{~nm}, 0.5$ $\mathrm{nm}, 0.5 \mathrm{~nm}$ ) in space with reference to origin.

After mixing solute in solvent, the system needs to be energy minimized to avoid local strains which ap- pear due to the atomic overlapping. These strains produce unrealistically high forces among the atoms and our system will be in far-fromequilibrium state which causes our simulation to fail [26]. Steepest- descent algorithm is used for energy minimization, but the process stops as soon as the maximum force is less than some tolerance value and total potential energy is negative. The negative potential energy in calculations confirms that the system is energy minimized. However, to study solvation free energy change, energy minimization process is carried out twice keeping all other parameters and conditions same as that done for diffusion phenomenon. First with flexible bond to allow atoms to move away from each other in a controlled manner and second with constrained bond to make sure the new constrained positions are not experiencing large forces since overlapping causes large inter-atomic forces. The potential energy of the system after minimization is shown in Figure (2).

The system is brought in thermal and pressure equilibrium after energy minimization. The thermodynamic properties of the system vary with different parameters like temperature, pressure, density etc. Variations of these parameters would affect the accuracy of those thermodynamic properties to be calculated. Therefore, a system needs to be subjected to equilibration before production run [27]. The sucrose-water system is equilibrated at four different temperatures ranging from $298.15 \mathrm{~K}$ to $318.15 \mathrm{~K}$ and pressure of 1 bar with isothermal compressibility of $4.6 \times 10^{-5} / \mathrm{bar}$. Velocity rescale thermostat for temperature coupling and Berendsen barostat for pressure coupling are used. The coupling time constants for thermostat and barostat are, respectively, $0.01 \mathrm{ps}$ and $1.0 \mathrm{ps}$. The total equilibration run time is $100 \mathrm{~ns}$. The
Particle Mesh Ewald (PME) algorithm is used for long range interaction. The cut-off parameter of 1 $\mathrm{nm}$ is taken with periodic boundary conditions for coulomb and Lennard-Jones (LJ) interaction. The equilibration process for solvation free energy of sucrose in water was performed at 300 $\mathrm{K}$, keeping all other parameters same. The densities and simulated temperatures at different coupling temperatures are shown in table 3 . After equilibration, production run is carried out to calculate the thermodynamical properties of the system such as diffusion coefficient and solvation free energy using NVT ensemble. The velocityrescale thermostat is used for this run. We couple the system to a fix pressure using Parinellorahman scheme in calculation of solvation free energy. The production run for diffusion is performed for $100 \mathrm{~ns}$ with the time step of $2 \mathrm{fs}$ whereas for solvation free energy, the production run is completed in 100000 steps with 0.002 ps step size which takes $0.2 \mathrm{~ns}$ production run time. In solvation free energy production run, we use sd integrator instead of md to account for friction of solvent molecules and high velocity collisions that perturb the system. Moreover, some free energy parameters $(\lambda s)$ are also added. Soft-core parameters like sc-power, sc-sigma, and sc-alpha are used to be sure that we never get overlapping particles. The production run is completed for each $\lambda s$ and input files for each of them are generated by using shell-scripting code.

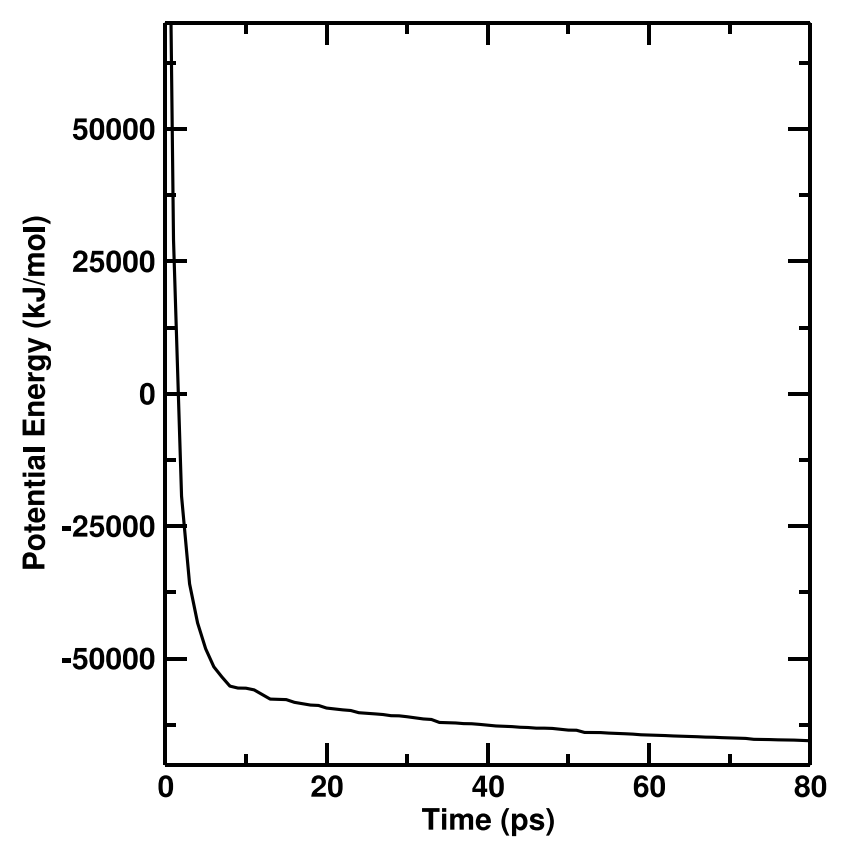

Fig. 2: Potential energy as a function of time after energy minimization. 
Table 3: Values of simulated temperature $\left(T_{\text {sim }}\right)$ and density at various coupling temperatures $\left(\boldsymbol{T}_{S U C}\right)$ for sucrose.

\begin{tabular}{lllll}
\hline S.N. & $\boldsymbol{T}_{\text {SUC }}(\mathbf{K})$ & $\boldsymbol{T}_{\text {sim }}(\mathbf{K})$ & $\boldsymbol{\rho}_{\text {system }}\left(\boldsymbol{k g} / \boldsymbol{m}^{\mathbf{3}}\right)$ & $\boldsymbol{\rho}_{\text {water }}\left(\boldsymbol{k g} / \boldsymbol{m}^{\mathbf{3}}\right)[28]$ \\
\hline 1. & 298.150 & $298.142 \pm 0.006$ & $999.122 \pm 0.031$ & 997.05 \\
\hline 2. & 303.150 & $303.143 \pm 0.003$ & $996.413 \pm 0.023$ & 995.65 \\
\hline 3. & 308.150 & $308.141 \pm 0.010$ & $993.648 \pm 0.024$ & 994.03 \\
\hline 4. & 318.150 & $318.135 \pm 0.003$ & $987.479 \pm 0.011$ & 992.59 \\
\hline
\end{tabular}

\section{RESULTS AND DISCUSSION}

\section{A. Radial Distribution Function (RDF)}

The radial distribution function gives the relative probability of finding a particle at a certain distance from a reference particle. From the reference particle to first near-neighbor shell, there is no chance of penetration of atoms (up to the distance of diameter of atoms), so there is no radial distribution function, and this region is regarded as exclusion region [29].

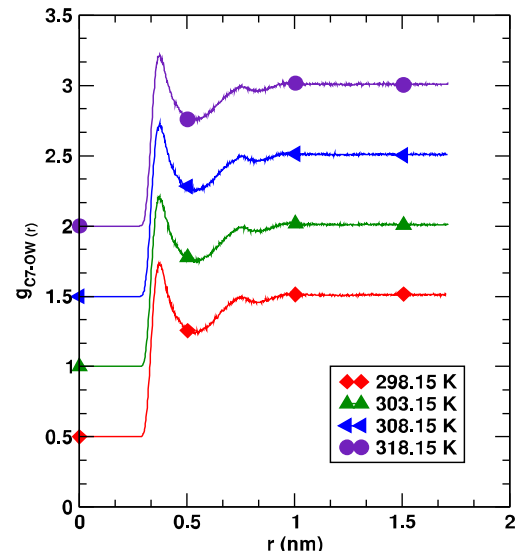

Fig. 3: RDF, g_(C7-OW) at different temperatures ranging from $298.15 \mathrm{~K}$ to $318.15 \mathrm{~K}$

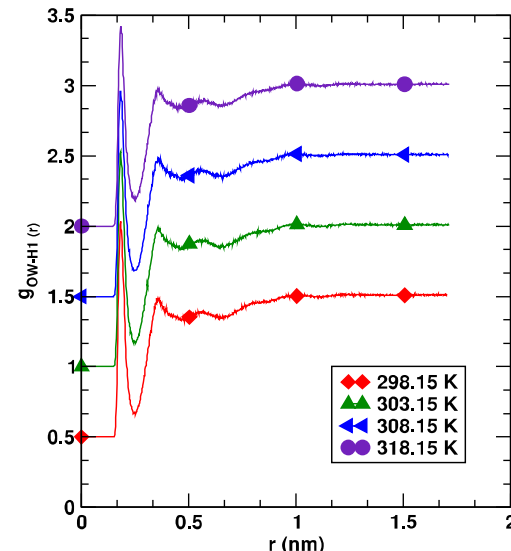

Fig. 4: RDF, g_(OW-H1) at different temperatures ranging from $298.15 \mathrm{~K}$ to $318.15 \mathrm{~K}$

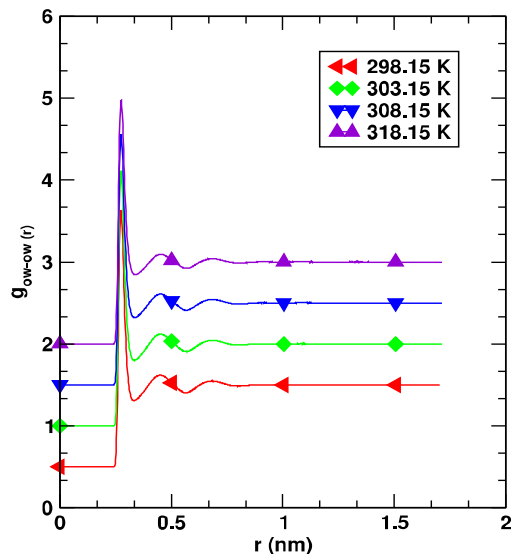

Fig. 5: RDF, g_(OW-OW) at different temperatures ranging from $298.15 \mathrm{~K}$ to $318.15 \mathrm{~K}$.
The RDFs of carbon (C7) and hydrogen (H1) atoms of sucrose molecules with reference to oxygen (OW) atoms of water are shown in figures (3) and (4) respectively. Similarly, the RDF of one of the oxygen atoms of water with reference to another oxygen atom of the same water is also plotted in figure (5). We can see the exclusion regions up to a certain distance, where the probability of finding the particle with respect to the reference particle is zero. As we go right side of each plot, the height of the peaks keeps on decreasing. This means that as the distance between the reference atom and the atom under study increases, the correlation between them decreases and eventually there won't be any long-range correlation for a large value of $r$ i.e., $g(r) \rightarrow 1$ [30].

Table 4: RDF, $g_{C 7-O W}$, analysis between carbon $(C 7)$ atom of sucrose molecule and oxygen atom $(\mathrm{OW})$ of water molecule.

\begin{tabular}{llllll}
\hline T $(\mathbf{K})$ & ER $(\mathbf{n m})$ & FPP $(\mathbf{n m})$ & FPV & SPP $(\mathbf{n m})$ & SPV \\
\hline $\mathbf{2 9 8 . 1 5}$ & 0.282 & 0.372 & 1.239 & 0.754 & 1.001 \\
\hline $\mathbf{3 0 3 . 1 5}$ & 0.280 & 0.372 & 1.234 & 0.756 & 0.995 \\
\hline $\mathbf{3 0 8 . 1 5}$ & 0.282 & 0.372 & 1.231 & 0.756 & 0.992 \\
\hline $\mathbf{3 1 8 . 1 5}$ & 0.278 & 0.372 & 1.220 & 0.758 & 0.991 \\
\hline
\end{tabular}


Table 5: RDF, $g_{O W-H 1}$, analysis between hydrogen $(\mathrm{H1})$ atom of sucrose molecule and oxygen atom (OW) of water molecule.

\begin{tabular}{llllll}
\hline T $(\mathbf{K})$ & \multicolumn{1}{c}{ ER $(\mathbf{n m})$} & FPP $(\mathbf{n m})$ & FPV & SPP $(\mathbf{n m})$ & SPV \\
\hline $\mathbf{2 9 8 . 1 5}$ & 0.148 & 0.182 & 1.532 & 0.350 & 0.998 \\
\hline $\mathbf{3 0 3 . 1 5}$ & 0.148 & 0.180 & 1.514 & 0.356 & 0.991 \\
\hline $\mathbf{3 0 8 . 1 5}$ & 0.148 & 0.180 & 1.460 & 0.356 & 0.989 \\
\hline $\mathbf{3 1 8 . 1 5}$ & 0.146 & 0.182 & 1.424 & 0.358 & 0.971 \\
\hline
\end{tabular}

Table 6: RDF, $g_{O W-O W}$, analysis between oxygen atoms (OW-OW) of water molecules.

\begin{tabular}{clllllll}
\hline T $(\mathbf{K})$ & ER $(\mathbf{n m})$ & FPP $(\mathbf{n m})$ & FPV & SPP $(\mathbf{n m})$ & SPV & TPP $(\mathbf{n m})$ & TPV \\
\hline $\mathbf{2 9 8 . 1 5}$ & 0.240 & 0.274 & 3.130 & 0.450 & 1.121 & 0.686 & 1.048 \\
\hline $\mathbf{3 0 3 . 1 5}$ & 0.240 & 0.276 & 3.105 & 0.450 & 1.121 & 0.690 & 1.045 \\
\hline $\mathbf{3 0 8 . 1 5}$ & 0.240 & 0.276 & 3.054 & 0.450 & 1.111 & 0.690 & 1.043 \\
\hline $\mathbf{3 1 8 . 1 5}$ & 0.240 & 0.276 & 2.976 & 0.456 & 1.095 & 0.690 & 1.040 \\
\hline
\end{tabular}

The heights and positions of the peaks for figures (3), (4), and (5) are provided in tables (4), (5), and (6) respectively, where $\mathrm{ER}=$ Exclusion region, FPP $=$ First Peak Point, FPV $=$ First Peak Value, SPP $=$ Second Peak Point, SPV $=$ Second Peak Value, TPP $=$ Third Peak Point, TPV $=$ Third Peak Value. For H1, C7, and OW atoms, the exclusion regions extend up to $0.148 \mathrm{~nm}, 0.282 \mathrm{~nm}$, and $0.240 \mathrm{~nm}$ respectively at all temperatures. In figures (3) and (4), the roughness in the curve is due to insufficient statistics caused by fewer number of $\mathrm{C} 7$ and $\mathrm{H} 1$ atoms of sucrose. Moreover, from tables (4-6), we can also see that as we increase the temperature, the height of the peak

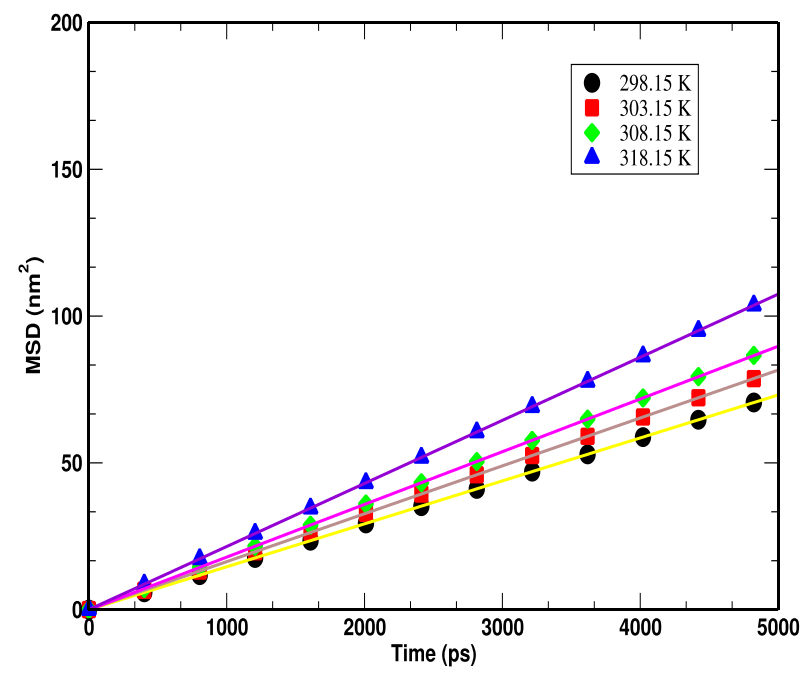

Fig. 6: MSD vs time plot of water molecule at different temperatures. decreases, which means that the atoms get more scattered and less structured as we increase the temperature.

\section{B. Diffusion Coefficients}

The in-built GROMACS package $g m x m s d$ is used to calculate the self-diffusion coefficients. This package generates a data file of average meansquared displacement as a function of time. By performing a linear fit of this data, we can obtain the slope of the straight line. As required by Einstein's relation, dividing the slope by the factor 6 , we get self-diffusion coefficient.

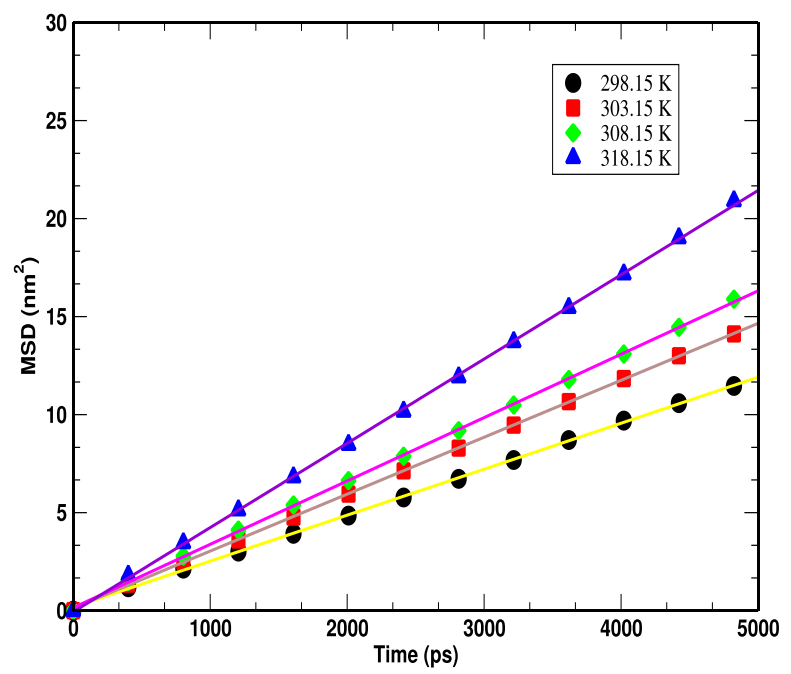

Fig. 7: MSD vs time plot of sucrose molecule at different temperatures. 
The figures (6) and (7) show the MSD plots of sucrose and water molecules at temperatures ranging from $298.15 \mathrm{~K}$ to $318.15 \mathrm{~K}$ respectively. The corresponding simulated results of self-diffusion coefficients are shown in tables (7) and (8) respectively. It is observed that when temperature increases, the self-diffusion coefficient also increases. This is because, as the temperature increases, the generated velocities of the sucrose and water molecules also increase and the density of the system decreases. These provide more space for the molecules to execute random walk. Due to this, the mean-squared displacement increases. By Einstein's relation, as the mean-squared displacement increases, the self-diffusion coefficient also increases. Comparing the simulated results of selfdiffusion coefficients of water with the experimental values, the results are within $5.84 \%$ error.

Table 7: Simulated values of self-diffusion coefficient of sucrose at different temperatures.

\begin{tabular}{rc}
\hline $\mathbf{T}(\mathbf{K})$ & $\begin{array}{c}\text { Self-Diffusion Coefficient } \\
\left(\times \mathbf{1 0}^{-\mathbf{9}} \boldsymbol{m}^{\mathbf{2}} \boldsymbol{s}^{\mathbf{1}}\right)\end{array}$ \\
\hline $\mathbf{2 9 8 . 1 5}$ & $0.390883 \pm 0.000013$ \\
\hline $\mathbf{3 0 3 . 1 5}$ & $0.484000 \pm 0.000067$ \\
\hline $\mathbf{3 0 8 . 1 5}$ & $0.538700 \pm 0.000015$ \\
\hline $\mathbf{3 1 8 . 1 5}$ & $0.716450 \pm 0.000024$
\end{tabular}

Table 8: Simulated and experimental values of self-diffusion coefficient of water at different temperatures.

T (K) Simulated $\left(\times 10^{-9} \mathrm{~m}^{2} \mathrm{~s}^{-1}\right) \quad$ Experimental $\left(\times 10^{-9} \mathrm{~m}^{2} \mathrm{~s}^{-1}\right)[31] \quad \%$ Error

\begin{tabular}{llll}
\hline $\mathbf{2 9 8 . 1 5}$ & $2.43833 \pm 0.00006$ & 2.30380 & 5.84 \\
\hline $\mathbf{3 0 3 . 1 5}$ & $2.71867 \pm 0.00003$ & 2.59960 & 4.58 \\
\hline $\mathbf{3 0 8 . 1 5}$ & $2.99217 \pm 0.00009$ & 2.91570 & 2.62 \\
\hline $\mathbf{3 1 8 . 1 5}$ & $3.58150 \pm 0.00003$ & 3.60700 & 0.70 \\
\hline
\end{tabular}

The binary diffusion coefficients are calculated by using Darken's relation. The mole fractions of sucrose and water are taken as $1.49 \times 10^{-3}$ and
0.9985 respectively. Table (9) shows the simulated and experimental results of the binary diffusion coefficients of sucrose.

Table 9: Simulated and experimental values of binary diffusion coefficient of sucrose in water at different temperatures.

\begin{tabular}{llll}
\hline $\mathbf{T}(\mathbf{K})$ & Simulated $\left(\times \mathbf{1 0}^{-\mathbf{9}} \boldsymbol{m}^{\mathbf{2}} \boldsymbol{s}^{\mathbf{- 1}}\right)$ & Experimental $\left(\times \mathbf{1 0}^{-\mathbf{9}} \boldsymbol{m}^{\mathbf{2}} \boldsymbol{s}^{\mathbf{1}}\right)[\mathbf{9}]$ & \% Error \\
\hline $\mathbf{2 9 8 . 1 5}$ & $0.39400 \pm 0.00010$ & $0.50400 \pm 0.00400$ & 21.83 \\
\hline $\mathbf{3 0 3 . 1 5}$ & $0.48700 \pm 0.00011$ & $0.59400 \pm 0.01700$ & 18.01 \\
\hline $\mathbf{3 0 8 . 1 5}$ & $0.54200 \pm 0.00005$ & $0.69200 \pm 0.00700$ & 21.67 \\
\hline $\mathbf{3 1 8 . 1 5}$ & $0.72100 \pm 0.00009$ & $0.88800 \pm 0.01200$ & 18.80 \\
\hline
\end{tabular}

\section{Solvation Free Energy}

A bar graph of change in free energies for each successive values of $\lambda$ is shown in figure (8). Each bar gives the change in free energy $(\Delta G)$ between neighboring values of $\lambda$. For an example, for $\lambda=0.2$, the change in free energy is the sum of free energy difference between $\lambda=0.0 \& 0.2$ and $\lambda=0.4 \& 0.2$.

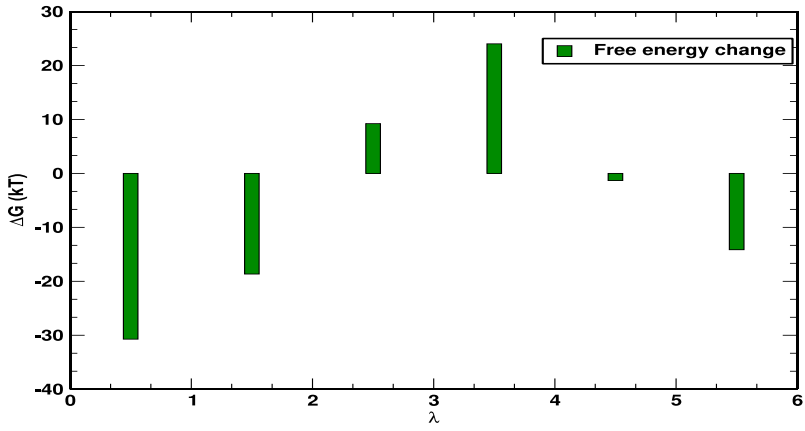

Fig. 8: Change in free energy for each successive value of $\lambda$. 
Table (10) shows the values of free energy change between each value of $\lambda$ and the total solvation free energy of the system is found to be $-78.92 \pm 12.77$ $\mathrm{kJ} / \mathrm{mol}$.

Table 10: Details of free energy change between each neighboring value of $\lambda$ and total solvation free energy of the system at $300 \mathrm{~K}$.

\begin{tabular}{llll}
\hline S.N. & Point & \multicolumn{1}{c}{$\boldsymbol{\lambda}$} & \multicolumn{1}{c}{$\boldsymbol{\Delta G}$} \\
\hline $\mathbf{1 .}$ & $0 \rightarrow 1$ & 0.0 & $-76.66 \pm 0.26$ \\
\hline 2. & $1 \rightarrow 2$ & 0.2 & $-46.55 \pm 0.32$ \\
\hline $\mathbf{3 .}$ & $2 \rightarrow 3$ & 0.4 & $22.99 \pm 1.77$ \\
\hline $\mathbf{4 .}$ & $3 \rightarrow 4$ & 0.6 & $59.91 \pm 4.97$ \\
\hline $\mathbf{5 .}$ & $4 \rightarrow 5$ & 0.8 & $-3.32 \pm 1.11$ \\
\hline $\mathbf{6 .}$ & $5 \rightarrow 6$ & 0.9 & $-35.29 \pm 4.34$ \\
\hline 7. & Total & $0.0 \rightarrow 1.0$ & $-78.92 \pm 12.77$ \\
\hline
\end{tabular}

\section{Temperature Dependence}

So far, it has been observed that the diffusion coefficient depends on temperature. This temperature dependency can be explained by Arrhenius equation [9]

$$
D=D_{0} e^{-\frac{E_{\alpha}}{N_{A} k_{B} T}}
$$

$D_{0}$ is pre-exponential factor, which is also called frequency factor, $E_{\alpha}$ is activation energy for diffusion and $N_{A}$ is Avogadro's number. This equation can further be written as,

$$
E_{\alpha}=-N_{A} k_{B} \frac{\partial \ln D}{\partial(1 \backslash T)}
$$

Taking the slope of $\ln D$ vs $1 \backslash T$ graph and multiplying with $-N_{A} k_{B}$, activation energy can be calculated. Figure (9) shows the Arrhenius plot for the binary diffusion coefficient of sucrose in water for both experimental and simulation results.

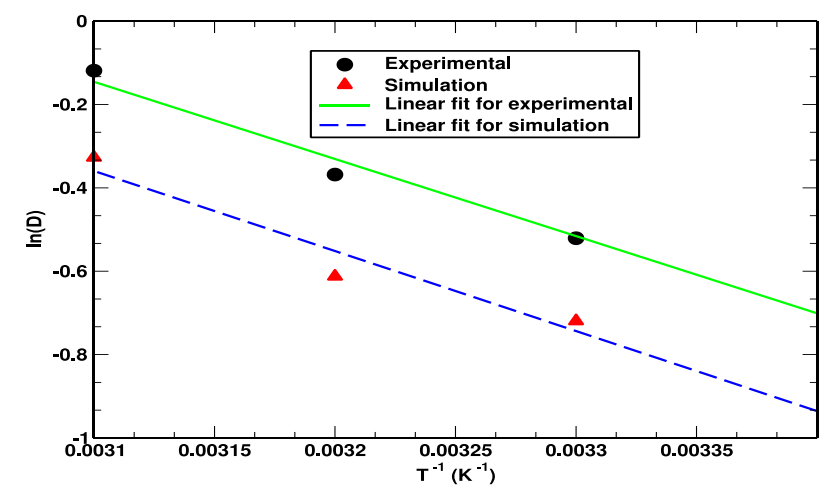

Fig. 9: Arrhenius diagram for the simulated and experimental values of binary mixture of sucrose and water.
If we extrapolate the graph to $\frac{1}{T} \rightarrow 0$, we can evaluate the pre-exponential factor $D_{0}$. The activation energy from the simulation was found to be $15.96 \mathrm{~kJ} / \mathrm{mol}$. This gives the amount of energy required to diffuse the sucrose in water.

\section{CONCLUSION AND FUTURE PROSPECTS}

In this work, we have carried out a classical molecular dynamics simulation with 1343 molecules of SPC/E model water and 2 molecules of sucrose at $298.15 \mathrm{~K}$, 303.15 K, 308.15 K, 318.15 K temperatures. The molar concentration of sucrose in water is 0.08 $\mathrm{mol} / \mathrm{m}^{3}$ for all temperatures. The density and temperature fluctuations have been studied to know the equilibrated temperature and density of the system. Self-diffusion and binary diffusion coefficients have shown a good agreement with the previously reported experimental results. The effect of temperature on equilibrium structure of the system has been observed with the help of radial distribution function. It is observed that when we increase temperature, the system becomes more random. The relationship between diffusion coefficient and temperature has been clarified by using Arrhenius graph. The solvation free energy of sucrose at $300 \mathrm{~K}$ has been estimated by using free energy perturbation method. However, the error in its calculation is a bit high, which is due to the insufficient number of intermediate states. Thus, we conclude that molecular dynamics simulation is one of the best tools to study equilibrium structure, transport properties, solvation free energy and other properties of biomolecules with low computational cost. The results given by this technique are very close to the results obtained from experiment, so we can consider this as a reliable tool for a good researcher.

In future, we intend to extend this work in various ways. We aim to calculate diffusion coefficients by taking a constant temperature with varying concentration of the solute. We are looking forward to using different water models like TIP3P, TIP4P etc. or different organic solvents like ethanol. Further, we set a goal to calculate and compare the diffusion coefficients using velocity autocorrelation function (VACF) and Einstein's relation. Moreover, we plan to calculate solvation free energy of sucrose at various temperatures.

\section{REFERENCES}

[1] Nelson, D. L.; and Cox, M. M., Lehinger Principles of Biochemistry, p. 238, W.H. Freeman (2004). 
[2] Vlitos, A. J., Sucrose: Properties and Applications, p. 186-222, Chapman and Hall, New York (1995).

[3] Colonna, W. J.; Samaraweera, U.; Clarke, M. A.; Cleary, M.; Godshall, M. A.; and White, J. S.; Encyclopedia of Chemical Technology, 19: 2-3, John Wiley \& Sons (2006).

[4] Cooper, J. M.; Optimizing sweet taste in foods, p. 135, Woodhead Publishing Limited, England (2006).

[5] Navarro, S. Z.; and Llamas, F. P. The importance of sucrose for cognitive functions, knowledge, and behaviour, Nutricion Hospitalaria, 28 (4): 106111 (2013).

[6] Rippe, J. M.; and Angelopoulos, T.J. Relationship between added sugars consumption and chronic disease risk factors: current understanding, Nutrients, 8 (11): 697 (2016).

[7] Pokharel, S.; Pantha, N.; and Adhikari, N. P. Diffusion coefficient of nitric oxide in water: a molecular dynamics study, International Journal of Modern Physics, 30 (27): 1650205 (2016).

[8] Price, H. C.; Mattsson, J.; and Murray, B. J. Sucrose diffusion in aqueous solution, Physical Chemistry Chemical Physics, 18: 19207-19216 (2016).

[9] Ribiero, A. C. F.; Ortona, O.; Simoes, S. M. N.; Santos, C. I. A. V.; Prazeres, P. M. R. A.; Valente, A. J. M.; Lobo, V. M. M.; and Burrows, H.D. Binary mutual diffusion coefficients of aqueous solution of Sucrose, Lactose, Glucose, and Fructose in the temperature range from (218.15 - 328.15) K, Journal of Chemical \& Engineering Data, 51 (5): 1836-1840 (2006).

[10] Skyner, R. E.; McDonagh, J. L.; Groom, C. R.; Mourik, T. v.; and Mitchell, J. B. O. A review of methods for the calculation of solution free energies and modelling of systems in solution, Physical Chemistry Chemical Physics, 17: 6174-6191 (2015).

[11] Shirts, M. R.; Pitera, J. W.; Swope, W. C.; and Pande, V. S. Extremely precise free energy calculations of amino acid side chain analogs: Comparison of common molecular mechanics force fields for proteins, The Journal of Chemical Physics, 119 (11): 5740-5761 (2003).

[12] Shirts, M. R.; and Pande, V. S. Comparison of efficiency and bias of free energies computed by exponential averaging, the Bennett acceptance ratio, and thermodynamic integration, The Journal of Chemical Physics, 122: 144107 (2005).

[13] Heitjans, P.; and Karger, J., Diffusion in Condensed Matter: Methods, Materials, Models, p. 249, Springer, Berlin (2005).

[14] Frankel, D.; and Smit, B., Understanding Molecular Simulation: from algorithms to applications, 1, Academic Press (2002).

[15] Malenkov, G. Liquid water and ices: understanding the structure and physical properties, Journal of Physics: Condensed Matter, 21 (28): 283101 (2009).
[16] Darken, L. S. Diffusion, mobility and their interrelation through free energy in binary metallic systems, Transactions of AIME, 175: 184-201 (1948).

[17] Klimovich, P. V.; Shirts, M. R.; and Mobley, D. L. Guidelines for the analysis of free energy calculations, Journal of Computational Aided Molecular Design, 29 (5): 397-411 (2015).

[18] Paliwal, H.; and Shirts, M. R. A benchmark test set for alchemical free energy transformations and its use to quantify error in common free energy methods, Journal of Chemical Theory and Computation, 7(12): 4115-4134 (2011).

[19] Pohorille, A.; Jarzynski, C.; and Chipot, C. Good practices in free-energy calculations, Journal of Physical Chemistry B, 114 (32): 10235-10253 (2010).

[20] Haile, J. M., Molecular Dynamics simulation: elementary methods, p. 1, John Wiley \& Sons, New York (1992).

[21] Montecelli, L.; and Tieleman, D.P., Force fields for classical molecular dynamics, 924, Springer Science + Business Media, New York (2013).

[22] Bhusal, S.; and Pantha, N. Diffusion of fructose in water: a molecular dynamics study, Himalayan Physics, 9: 30-44 (2020)

[23] Thapa, S. K.; and Adhikari, N. P. A molecular dynamics study of oxygen gas in water at different temperatures, International Journal of Modern Physics B, 27 (8): 1350023 (2013).

[24] van der Spoel, D.; Van Mareen, P. J.; and Berendsen, H. J. C. A systematic study of water models for molecular simulation: derivation of water models optimized for use with a reaction field, The Journal of Chemical Physics, 108 (24): 10220-10230 (1998).

[25] Berendsen, H. J. C.; Grigera, J. R.; and Straatsma, T. P. The missing term in effective pair potentials, Journal of Physical Chemistry, 91: 6269-6271 (1987).

[26] Poudyal, I.; and Adhikari, N. P. Temperature dependence of diffusion coefficient of carbon monoxide in water: A molecular dynamics study, Journal of Molecular Liquid, 194: 77-84 (2014).

[27] Koirala, R. P.; Dawanse, S.; and Pantha, N. Diffusion of glucose in water: a molecular dynamics study, Journal of molecular liquid, 345: 117826 (2022).

[28] “Density of water", accessed: 2020-08-05. https://antoine.frostburg.edu/chem/senese/javascri pt/water-density.html

[29] Chaikin, P. M.; and Lubensky, T. C., Principles of Condensed Matter Physics, p. 8-40, Cambridge University Press (1995).

[30] Chandler, D., Introduction to Modern Statistical Mechanics, Oxford University Press (1987).

[31] "Diffusion Coefficient of water", accessed: 202008-29. https://dtrx.de/od/diff 